\title{
Prediction of Young's Modulus for Injection Molded Long Fiber Reinforced Thermoplastics
}

\author{
Hongyu Chen ${ }^{1}$ and Donald G. Baird ${ }^{1,2, *}$ \\ 1 Department of Chemical Engineering, Virginia Tech, VA 24061, USA; hongyu86@vt.edu \\ 2 Macromolecules Innovation Institute, Virginia Tech, VA 24061, USA \\ * Correspondence: dbaird@vt.edu; Tel.: +1-540-231-5998
}

Received: 26 June 2018; Accepted: 2 August 2018; Published: 6 August 2018

\begin{abstract}
In this article, the elastic properties of long-fiber injection-molded thermoplastics (LFTs) are investigated by micro-mechanical approaches including the Halpin-Tsai (HT) model and the Mori-Tanaka model based on Eshelby's equivalent inclusion (EMT). In the modeling, the elastic properties are calculated by the fiber content, fiber length, and fiber orientation. Several closure approximations for the fourth-order fiber orientation tensor are evaluated by comparing the as-calculated elastic stiffness with that from the original experimental fourth-order tensor. An empirical model was developed to correct the fibers' aspect ratio in the computation for the actual as-formed LFTs with fiber bundles under high fiber content. After the correction, the analytical predictions had good agreement with the experimental stiffness values from tensile tests on the LFTs. Our analysis shows that it is essential to incorporate the effect of the presence of fiber bundles to accurately predict the composite properties. This work involved the use of experimental values of fiber orientation and serves as the basis for computing part stiffness as a function of mold filling conditions. The work also explains why the modulus tends to level off with fiber concentration.
\end{abstract}

Keywords: long glass fibers; elastic properties; fiber orientation; fiber length; fiber bundles

\section{Introduction}

During the last decade, an enhanced demand for lightweight materials in automotive applications has resulted in the growth of the use of thermoplastic-discontinuous fiber composites [1]. The increased growth of the use of these thermoplastic matrix composite systems is due to the combination of mechanical properties and melt processability. Long-fiber (lengths $>1 \mathrm{~mm}$ ) thermoplastic composites (LFTs) possess significant advantages over short fiber $(<1 \mathrm{~mm})$ composites in terms of their mechanical properties while retaining their ability to be injection molded [2]. The goal of this research is to improve the stiffness properties predictions for injection molded LFTs. During the plasticating stage of injection molding, significant fiber attrition will occur leading to a broad fiber length distribution (FLD) [3]. Fiber orientation distribution (FOD) is another highly anisotropic feature of the final injection molded parts induced by the mold filling process [4]. Mechanical properties of LFTs are highly dependent on these microstructural variables imparted by the injection molding process [5].

The computation of the elastic stiffness for the aligned and monodispersed short fiber composites was well studied by a large range of people. Tucker et al. [6] reviewed the micromechanical models for this type of composite. By comparing the standard micromechanical models with their finite element method, the authors have shown that the Halpin-Tsai equation gives reasonable estimates for stiffness, but the best predictions come from the Mori-Tanaka model based on the Eshelby's equivalent inclusion method. A similar conclusion was reached by Hine [7]. Ingber and Papathanasiou [8] found the variant of the Halpin-Tsai model is in very good agreement with their boundary element method (numerical simulation) for their entire range of fiber volume contents and aspect ratios, although they had no 
experimental data for comparison. These models for aligned and monodispersed fiber composites can predict the properties of a representative volume element that can subsequently be averaged to include effects of fiber length and orientation distributions of a real injection molded material. Hine et al. [7] carried out a numerical simulation using a distribution of fiber lengths generated by the Monte-Carlo technique. Garesci et al. [5] applied the fitted probability density function to get the averaged property from each single fiber length. A relatively concise way is to replace the FLD with some sort of mean fiber length $[7,9,10]$. The most widely used orientation-averaging scheme is to use second and/or fourth order orientation tensors developed by Tucker and Advani [4] to average the property constants. Hine et al. [11] have shown that the results determined by the constant strain orientation averaging method (assuming the units have the same strain and average their stiffness constants) were in good agreement with their numerical simulations.

There exists very little modeling work for predicting stiffness properties on injection molded LFTs [5]. The scenario of the LFTs is different from the works that studied short fiber composite. LFTs injection molding pellets prepared by the pultrusion technique have received much attention. In particular, the fibers are in the form of aligned fiber bundles coated by thermoplastic matrix, which produces pellets with much higher fiber contents than those of more conventional 'short-fiber' compounds. During the compounding process, filamentization of fiber bundles and fracture of the resultant monofilaments into elements of a lower aspect ratio lead to the dispersion of fibers into the polymer matrix [12]. For high content fiber composites, the presence of fiber bundles seems to be unavoidable which could result in a reinforcement with a much lower aspect ratio and effective stiffness than well-dispersed fibers, consequently giving a lower and even decreasing stiffness [13]. However, the existing stiffness models assume the fibers are fully and evenly dispersed in the matrix. The predicted values keep increasing with the fiber content, which is not true within commercial fiber concentration for injection molded LFTs $[13,14]$. In this paper, we report on the development of an empirical model to correct the fibers' aspect ratio for the actual as-formed LFTs with fiber bundles under high fiber content. After the correction, the analytical predictions show good agreement with the experimental stiffness values from tensile tests on the LFTs for the whole fiber content range investigated. Our analysis shows that it is essential to incorporate the effect of the presence of fiber bundles to accurately predict the composite properties.

\section{Analytical Modelling Details}

\subsection{Orientation}

A single rigid fiber can be represented by a unit vector, $\mathbf{p}$, parallel to the fiber's long axis. The average orientation state of a fiber composite can be descried by even-ordered structural tensors [4]. In this study, the second and fourth order orientation used in the modeling are defined as:

$$
\begin{gathered}
\mathbf{A}=\langle\mathbf{p} \mathbf{p}\rangle=\int \mathbf{p p} \psi(\mathbf{p}) \mathrm{d} \mathbf{p} \\
\mathbf{A}_{4}=\langle\mathbf{p p p p}\rangle=\int \mathbf{p p p p} \psi(\mathbf{p}) \mathrm{d} \mathbf{p}
\end{gathered}
$$

where $\psi(\mathbf{p})$ is the probability distribution function for orientation, and the bracket $\langle\cdot\rangle$ denotes the average quantity over a volume domain. The second order orientation tensor, $\mathbf{A}$, can be measured, while the fourth order orientation tensor, $\mathbf{A}_{4}$, can either be obtained from experiments or estimated in terms of $\mathbf{A}$ using various closure approximation methods [4]. In this study, several approximation closures are implemented to calculate $\mathbf{A}_{4}$, and the stiffness results evaluated from these approximations are compared with that obtained from the experimentally measured $\mathbf{A}_{4}$. 


\subsection{Fiber Length}

Due to the compounding process, the injection molded LFTs will end up with a very broad fiber length distribution. The actual fiber length information can be described by the experimentally obtained probability of finding a fiber with length $l_{i}$ given by:

$$
p l\left(l_{i}\right)=\frac{N_{i}}{\sum N_{i}}
$$

where, $N_{i}$ is the measured number of fibers with length $l_{i}$. A more concise approach is to replace the FLD with a single length, normally the number or weight average length defined, respectively, as:

$$
\begin{aligned}
L_{n} & =\frac{\sum N_{i} l_{i}}{\sum N_{i}} \\
L_{w} & =\frac{\sum N_{i} l_{i}^{2}}{\sum N_{i} l_{i}}
\end{aligned}
$$

\subsection{Elastic Properties}

As mentioned in the introduction, both the Halpin-Tsai (HT) and Eshelby-Mori-Tanaka (EMT) methods are extensively studied for the unidirectional, or short fiber reinforced composites. The Halpin-Tsai method is the most widely used micromechanical model because of its ease of implementation $[15,16]$. The corresponding equations are derived from the self-consistent ideas of Hill [17], and the final implementation is semi-empirical in nature. Several authors have concluded that a constant strain assumption works better than the constant stress assumption, that is to say, stiffness averaging surpasses the compliance averaging in the computation [11,18]. So in this article, the HT equations are used to predict the compliance matrix, and then the stiffness matrix is obtained from the inverse of the compliance matrix. Finally, the material property is calculated by averaging the stiffness constants based on FLD and FOD.

In this study, the EMT method is also used and compared with the HT method. The EMT method is a combination of Eshelby's equivalent inclusion method and Mori-Tanaka's back stress analysis, and so, this model is valid even for large volume fraction of fibers. In particular, the equivalent inclusion method of Eshelby is applied in the computation of energy-release rate in terms of the equivalent eigenstrains defined in the fiber and crack [19]. As a result, the EMT method provides the overall stiffness of the composite weakened by fiber-end cracks.

To include the FLD effect on the elastic properties, we use the EMT or HT method to calculate the stiffness of a 'reference' unidirectional fiber composite using either the experimental fiber length probability $p l\left(l_{i}\right)$ :

$$
C_{i j k l}=\frac{\sum C_{i j k l}^{*}\left(l_{i} / d\right) * p l\left(l_{i}\right)}{\sum p l\left(l_{i}\right)}
$$

or using an average fiber length $L_{a v g}\left(L_{n}\right.$ or $\left.L_{w}\right)$ evaluated from the FLD:

$$
C_{i j k l}=C_{i j k l}^{*}\left(L_{a v g} / d\right)
$$

where $C_{i j k l}$ is the stiffness matrix having a specific fiber aspect ratio $l_{i} / d$ or $L_{a v g} / d$, and $d$ is the single fiber diameter.

To get the stiffness of the actual injection molded LFTs, a mean tensor averaging procedure is used [20]. Specifically, the stiffness of the calculated unidirectional fiber composite including the FLD effect is averaged over the as-formed fiber orientation state as follow: 


$$
\begin{gathered}
\bar{C}_{i j k l}=B_{1} A_{i j k l}+B_{2}\left(A_{i j} \delta_{k l}+A_{i j} \delta_{k l}\right)+B_{3}\left(A_{i k} \delta_{j l}+A_{i l} \delta_{j k}+A_{j l} \delta_{i k}+A_{j k} \delta_{i l}\right) \\
+B_{4} \delta_{i j} \delta_{k l}++B_{5}\left(\delta_{i k} \delta_{j l}+\delta_{i l} \delta_{j k}\right)
\end{gathered}
$$

where $\delta_{i j}$ denotes the Kronecker delta and the scalar parameters $B_{i}(i=1-5)$ are the invariants of the stiffness tensor of the calculated unidirectional fiber composite given as:

$$
\begin{gathered}
B_{1}=C_{11}+C_{22}-2 \times C_{12}-4 \times C_{66} \\
B_{2}=C_{12}-C_{23} \\
B_{3}=C_{66}+\frac{1}{2}\left(C_{23}-C_{22}\right) \\
B_{4}=C_{23} \\
B_{5}=\frac{1}{2}\left(C_{22}-C_{23}\right)
\end{gathered}
$$

The composite specimen can be considered as a stacking sequence of thin layers which might have a different fiber orientation state. The stiffness of each layer is calculated based on the overall FLD and the characterized orientation state using the above-mentioned method. Finally, the classical lamination theory [21] is then applied to calculate the overall effective engineering stiffness of the composite [20].

\section{Materials and Methods}

The composites under investigation were $10 \mathrm{wt} \%, 30 \mathrm{wt} \%, 40 \mathrm{wt} \%$, and $50 \mathrm{wt} \%$ glass fibers in a polypropylene matrix. The material was received from SABIC Innovative Plastics (Ottawa, IL, USA) as $12.5 \mathrm{~mm}$ long pellets created through a pultrusion process in $30 \mathrm{wt} \%$ and $50 \mathrm{wt} \%$ formulations. Samples with $10 \mathrm{wt} \%$ fibers were diluted with neat polypropylene, while $30 \mathrm{wt} \%$ and $50 \mathrm{wt} \%$ pellets were used to create the $40 \mathrm{wt} \%$ composites. The pellets contain a unidirectional bundle of fibers that must be dispersed during the injection molding process, specifically in the plasticating unit. Center-gated disk geometries were formed by injection molding as shown in Figure 1. In this study, the Hele-Shaw region (60\% disk radius) and the advancing front region ( $85 \%$ disk radius) were investigated for fiber length, fiber orientation, and mechanical properties. Tensile specimens were cut from the injection-molded samples, and the young's modulus was measured according to ASTM D3039 [22] for polymer matrix composite materials.

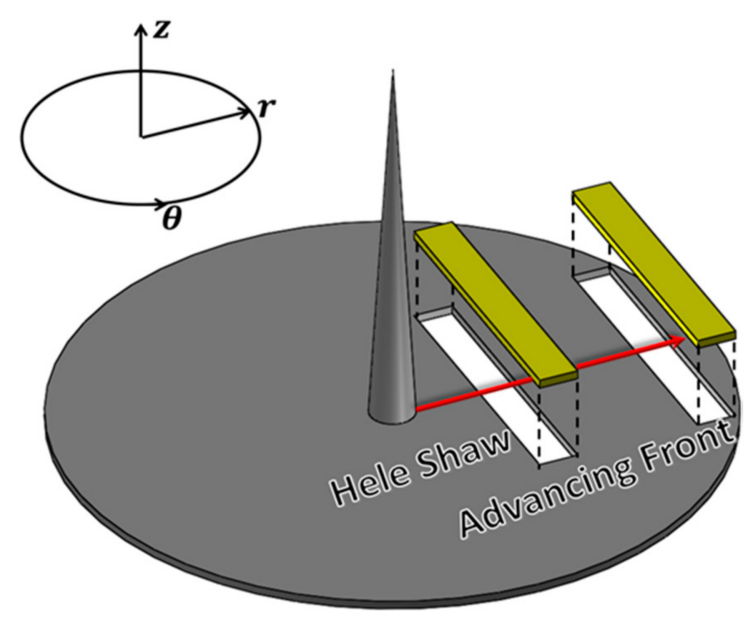

Figure 1. The injection-molded glass / ppcenter-gated disk: The Hele-Shaw region (60\% disk radius) and advancing front region ( $85 \%$ disk radius) were investigated in this study.

For fiber length measurement, a method based on Reference [23] was modified as follows: Instead of directly injecting epoxy into the samples after the burning off of the polymer matrix, a needle 
coated with epoxy was inserted into the sample all the way through the thickness direction shown in Figure 2a. As a result, fibers at this location were collected by the epoxy on the needle as shown in Figure $2 \mathrm{~b}$. Due to this sampling method, there was biased toward longer fibers, and the measured fiber length was then corrected based on the length of each fiber and the diameter of the needle with epoxy coating. The needle with the fibers was re-burned to get rid of the epoxy. Loose glass fibers were then dispersed on a desktop scanner and imaged at $3200 \mathrm{dpi}$. At least 3000 fibers per sample were measured using our in-house developed MATLAB ${ }^{\circledR}$ codes. The fiber length distribution followed the typical log-normal distribution commonly observed for fiber composites. Three samples were used to produce the averages in Table 1 . The number average $\left(L_{n}\right)$ and weight average $\left(L_{w}\right)$ fiber lengths were calculated according to Equations (4) and (5), respectively. It can be seen that the average fiber lengths in these samples are reduced with increasing fiber content.
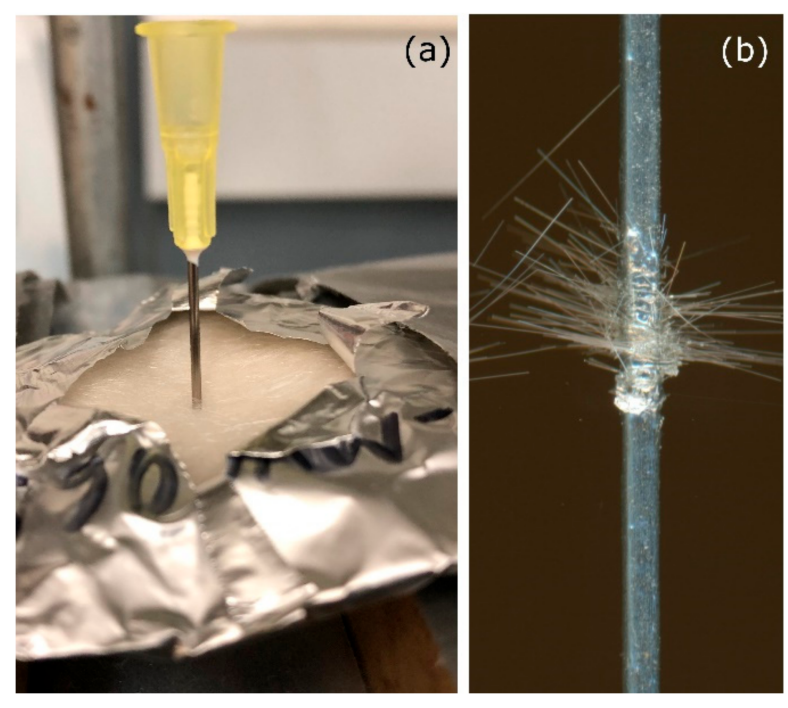

Figure 2. The modified fibers sampling method: (a) A needle coated with epoxy inserted into the desired location, and (b) the pulled out needle with the fibers attached on the surface of the epoxy.

Table 1. Fiber lengths information.

\begin{tabular}{ccccc}
\hline \multirow{2}{*}{ Fiber Content } & \multicolumn{2}{c}{ Hele-Shaw Region } & \multicolumn{2}{c}{ Advancing-Front Region } \\
\cline { 2 - 5 } & $\boldsymbol{L}_{\boldsymbol{n}}(\mathbf{m m})$ & $\boldsymbol{L}_{\boldsymbol{w}}(\mathbf{m m})$ & $\boldsymbol{L}_{\boldsymbol{n}}(\mathbf{m m})$ & $\boldsymbol{L}_{\boldsymbol{w}}(\mathbf{m m})$ \\
\hline $10 \mathrm{wt} \%\left(\mathrm{v}_{f}=0.038\right)$ & $1.51 \pm 0.081$ & $3.59 \pm 0.71$ & $1.76 \pm 0.12$ & $3.21 \pm 0.37$ \\
$30 \mathrm{wt} \%\left(\mathrm{v}_{f}=0.135\right)$ & $1.14 \pm 0.078$ & $3.41 \pm 0.41$ & $1.32 \pm 0.063$ & $3.52 \pm 0.26$ \\
$40 \mathrm{wt} \%\left(\mathrm{v}_{f}=0.197\right)$ & $0.98 \pm 0.080$ & $2.67 \pm 0.28$ & $1.03 \pm 0.086$ & $2.81 \pm 0.35$ \\
$50 \mathrm{wt} \%\left(\mathrm{v}_{f}=0.268\right)$ & $0.87 \pm 0.061$ & $2.42 \pm 0.32$ & $0.882 \pm 0.062$ & $2.54 \pm 0.21$ \\
\hline
\end{tabular}

Measurements of fiber orientation were also made to further investigate its relationship to the stiffness performance at the same locations. Orientation measurements were taken along the $r-z$ plane, such that $r$ denotes the flow direction with the velocity gradient in $z$. Samples were polished using modified metallographic techniques and oxygen plasma etched to enhance the contrast of the glass fiber and polypropylene matrix. Details of the sample preparation and orientation measurement procedure can be found in the References [24,25]. Figure 3 shows the measured through thickness fiber orientation for various glass fiber concentrations at the Hele-Shaw region. For the fiber orientation tensor, A, the diagonal components are the most important. They describe the alignment of the population with respect to the axis of the coordinate system. A value that approaches one indicates increased alignment in that direction. Only the $\theta$ direction component is presented here, because the young's modulus was measured along this transverse direction. At $30 \mathrm{wt} \%, 40 \mathrm{wt} \%$, and $50 \mathrm{wt} \%$, the through 
thickness fiber orientation distributions are very similar showing the characteristic shell-core-shell layer structures. Generally, the $\theta$ direction component reaches its largest value near the center of the disk, because that the center of the disk is dominated by extensional flow in this tangential direction. However, at $10 \mathrm{wt} \%$, the distribution deviates significantly from the rest. The through thickness fiber orientation distribution is relatively 'flat' compared to those with higher fiber concentrations. This might be due to the concentration effects on the fiber orientation dynamics. At $10 \mathrm{wt} \%$, the degree of fiber-fiber interaction is much less, that is to say, hindrance to fiber alignment is much less, as a result, the tangential direction alignment is quite dominant.

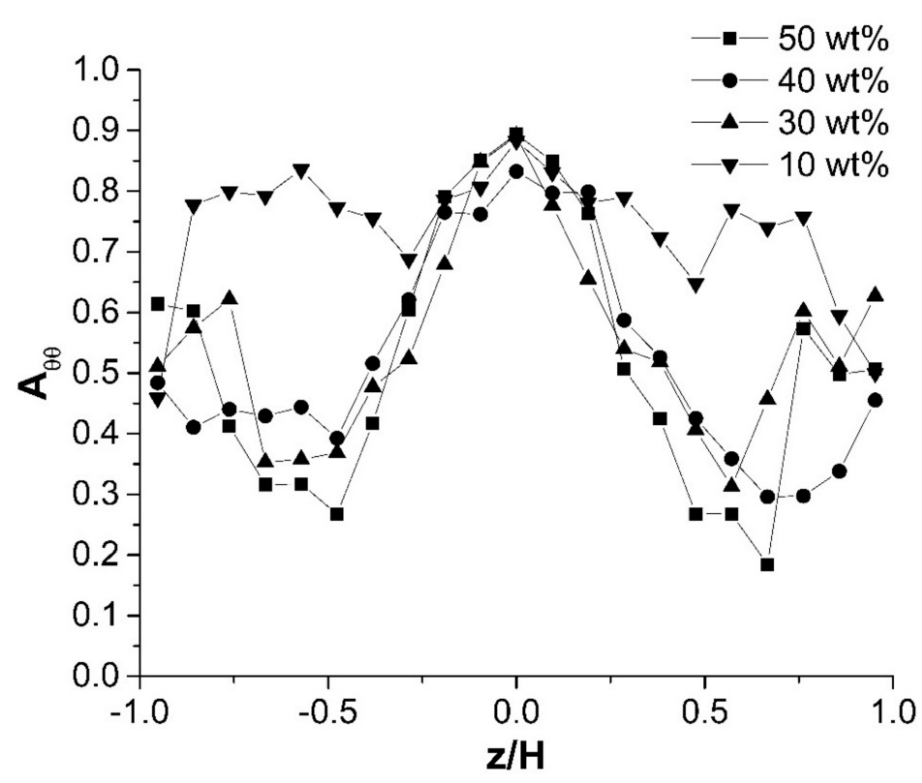

Figure 3. Measured $\theta$ direction fiber orientation distributions through the thickness direction for $10 \mathrm{wt} \%, 30 \mathrm{wt} \%, 40 \mathrm{wt} \%$ and $50 \mathrm{wt} \%$ glass fiber at the Hele-Shaw region.

\section{Results and Discussion}

In all the calculations in this article, the measured FLD or the corresponding average fiber length ( $L_{n}$ or $L_{w}$ ) was input into Equations (6) or (7), while the experimental second-order tensors were used in Equation (8). To analyze the accuracy of various closure approximations for predicting the elastic properties, the fourth order orientation tensors were evaluated by the linear (LIN), quadratic (QUA), hybrid (HYB), Invariant-based optimal fitting (IBOF), and improved orthotropic (ORW3) closure approximations $[4,18,26,27]$. Then, the as-calculated elastic stiffness using each of these estimated fourth order tensors was compared with that using the original experimental 'true' fourth-order tensor (TRU) obtained from Equation (2). The comparisons of the effective engineering modulus along the tangential direction are presented in Figures 4 and 5 applying the methods of EMT and HT, respectively. The predicted results and the general pattern with the HT and EMT methods in the studied fiber content range are very similar. Both models show a similar linear increase in the transverse modulus with increasing fiber content. However, all the values calculated from the EMT model, no matter what length parameter and closure approximation, are slightly greater than those from the HT model. Moreover, the differences between the two methods become more notable as fiber content increases. In Tucker and Liang's [6] review of the stiffness predictions for unidirectional fiber composites, for composites with an aspect ratio larger than 10, the EMT also has predicted greater values of the dominant modulus than the HT model. To answer the question which closure method or methods are the best for stiffness prediction purposes, the results calculated using the experimental fourth order orientation tensors are used as criteria. It seems that, for the entire fiber content range and all the scenarios using different fiber length parameters (FLD, $L_{n}$, and $L_{w}$ ), the magnitudes of IBOF and 
ORW3 predictions are the most comparable to the criteria. Another aspect of this paper is to examine the effects of the length parameters (FLD, $L_{n}$, and $L_{w}$ ) on the stiffness predictions for injection molded LFTs. It is seen that, the predictions of the $L_{n}$ parameters lie between the largest values generated by the $L_{w}$ parameters and the smallest predictions from the measured FLD. This result indicates that, for the purpose of replacing the FLD by an average fiber length in the computation, the $L_{n}$ might outperform the $L_{w}$ in terms of generating a better match with the FLD.
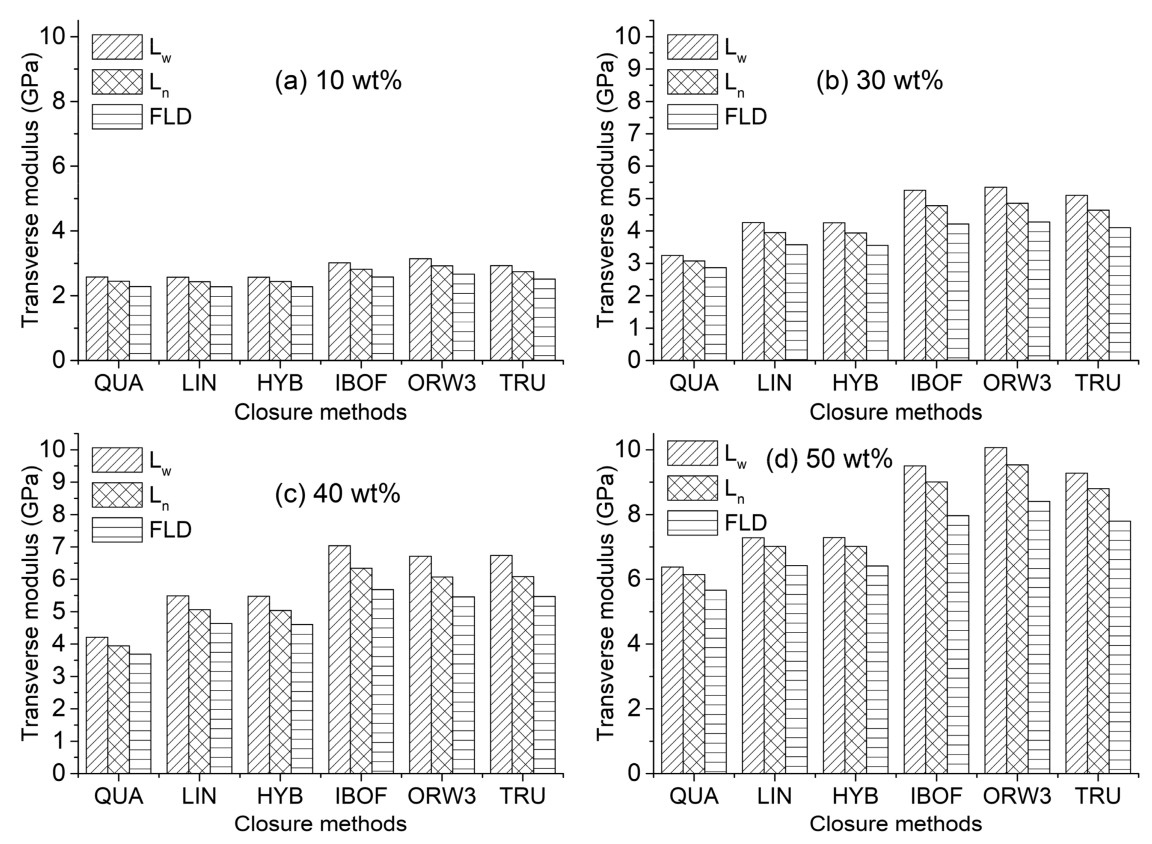

Figure 4. Predicted transverse modulus at the Hele-Shaw region using Eshelby-Mori-Tanaka (EMT) model for (a) $10 \mathrm{wt} \%$, (b) $30 \mathrm{wt} \%$, (c) $40 \mathrm{wt} \%$, and (d) $50 \mathrm{wt} \%$ glass fiber polypropylene composites. Various closure approximations and length parameters were used in the calculations.
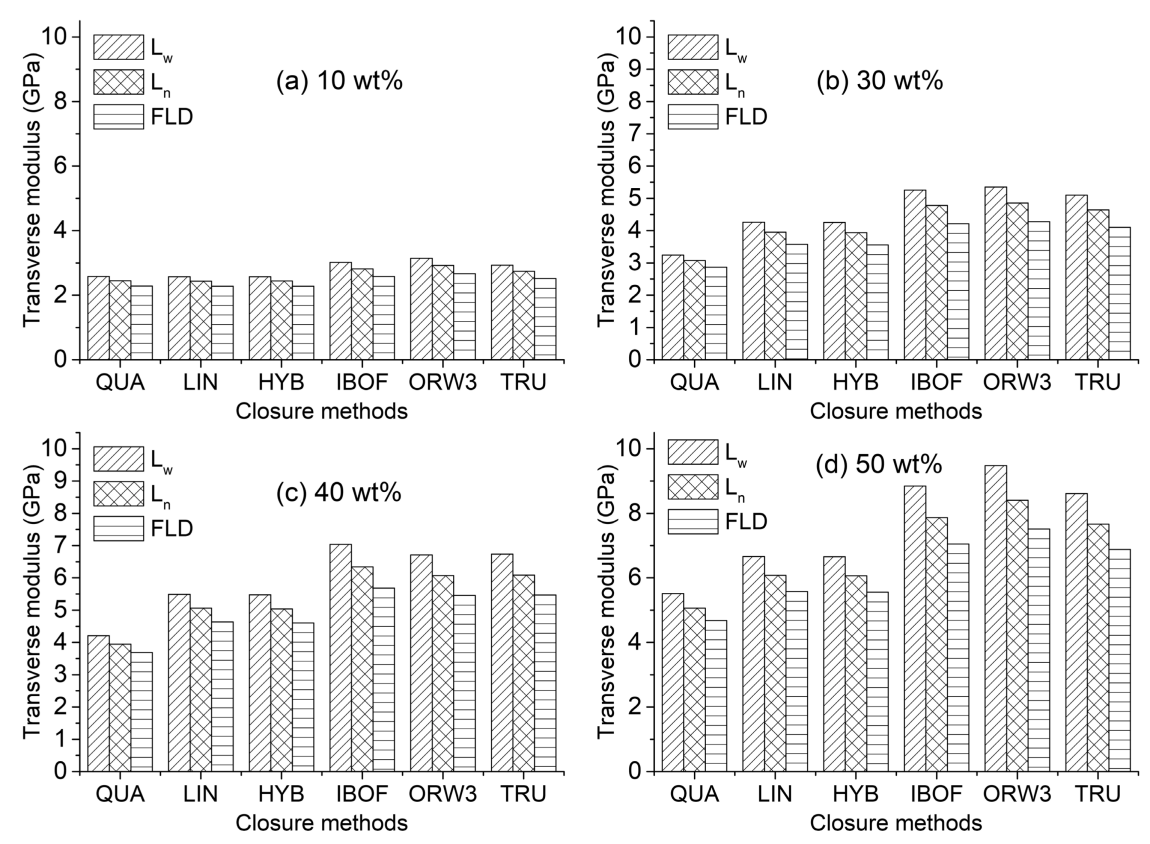

Figure 5. Predicted transverse modulus at the Hele-Shaw region using Halpin-Tsai (HT) model for (a) $10 \mathrm{wt} \%$, (b) $30 \mathrm{wt} \%$, (c) $40 \mathrm{wt} \%$, and (d) $50 \mathrm{wt} \%$ glass fiber polypropylene composites. Various closure approximations and length parameters were used in the calculations. 
The predictions are also compared with the transverse ( $\theta$ direction) tensile test results shown in Figure 6. At most locations through the thickness, the fibers are predominantly oriented toward this direction as shown in Figure 3. Here, both HT and EMT models are applied with the measured FLD and experimental fourth order orientation tensor. Only at the lowest $10 \mathrm{wt} \%\left(v_{f}=0.0385\right)$ fiber concentration do the predictions match well with the experimental results. As concentration increases, the deviations of the predictions from the experimental data turn out to be more significant. Several authors $[13,28]$ experimentally observed that any incremental increase in fiber content appears to bring a lower improvement in properties than the previous one. That is to say, the mechanical performance of the injection molded LFTs will reach a plateau or even decrease at very high fiber concentration range. There are several possible reasons for the degradation of the mechanical properties. First, due to the non-homogeneous nature of these materials, problems can arise during their manufacture, which result in void content/porosity in the final parts [29]. Second, there is also a possibility of poor adhesion between the glass fibers and the matrix [30]. Finally, at higher fiber content, fiber bundles are very common in the injection molded LFTs [13]. There are two major effects of the presence of fiber bundles on the mechanical performance of the composites. First, the clumping of fibers will reduce the effective fiber aspect ratios in the reinforcement. Second, fiber bundles have an effect on stress concentration. Specifically, the failed fiber will induce stress concentration in those un-failed neighbor fibers within the bundles. This stress concentration occurs during the nonlinear stage of the tensile test $[19,31]$. Therefore, it is valid to ignore stress concentration and exclusively consider the effects of reduced aspect ratio on modulus. In this study, the density of the $50 \mathrm{wt} \%$ injection molded samples was measured by the pycnometry method described in Reference [32]. The density given by the supplier is $1.33 \mathrm{~g} / \mathrm{mm}^{3}$ and the measured value of the injection molded center-gated-disk (CGD) is $1.327 \pm 0.0175 \mathrm{~g} / \mathrm{mm}^{3}$, which means the void content/porosity in the final parts is negligible. There is no information about the adhesion between the glass fibers and the matrix from the supplier. In this study, we assume the adhesion is perfect to simplify the problem, which most likely not be true. However, the $10 \mathrm{wt} \%, 30 \mathrm{wt} \%, 40 \mathrm{wt} \%$, and $50 \mathrm{wt} \%$ materials have the same surface treatments for the fibers (they are the same series using the same formulations). Therefore, it is legitimate to only include the effects of the clumping of fibers on the level-off of the elastic properties of the injection molded LFTs as fiber content increases.

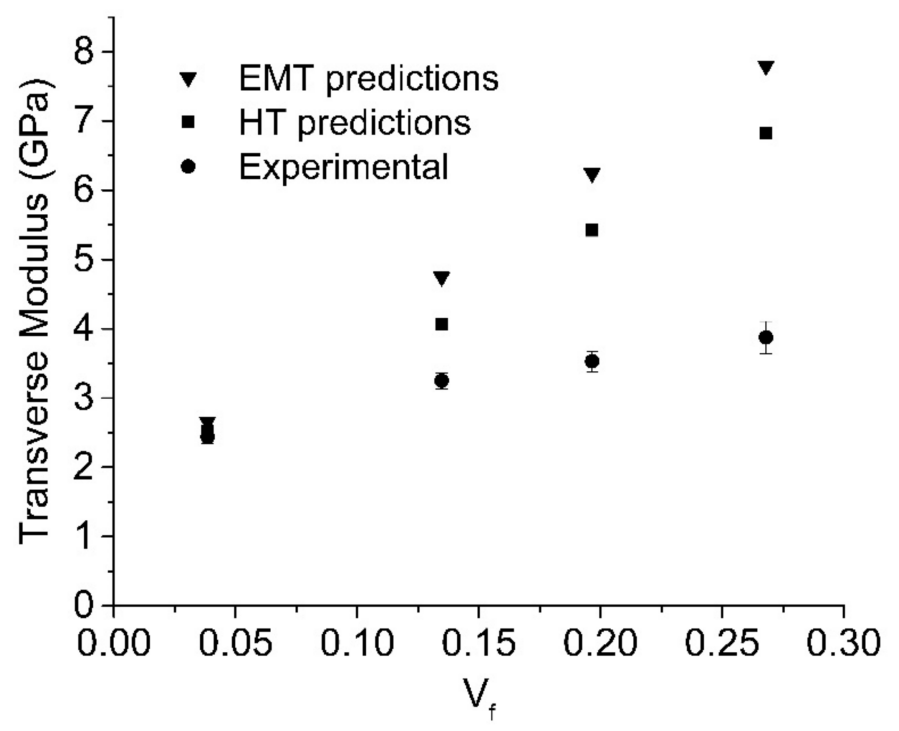

Figure 6. A comparison between the predictions using both HT and EMT methods, and the experimental tensile test results at various fiber content. 
The cross-sectional microscopic images on the $r-z$ plane with glass fiber foot-prints are shown in Figure 7. It is seen that the clumping of fibers turned out to be worse as fiber concentration increased. To include the effects of reduced aspect ratio on the modulus due to the existence of fiber bundles, an empirical model was proposed to modify the effective fiber bundle diameter $d_{c}$ in the stiffness computation.

$$
\begin{gathered}
d_{\text {effective }}=d_{0} \times d_{c}\left(l, \mathrm{v}_{f}\right) \\
d_{c}=1+\frac{\left(\frac{\sqrt{a_{r}}}{\exp \left(\frac{\mathrm{v}_{c}}{\mathrm{v}_{f}}\right)}\right)^{n}}{1+\exp \left(-\mathrm{v}_{f} /\left(1 / a_{r}\right)\right)} \\
a_{r}=\frac{l}{d_{0}}
\end{gathered}
$$

where, $d_{\text {effective }}$ is the effective bundle diameter, $d_{0}$ the single fiber diameter, $d_{c}$ a correction coefficient, $a_{r}$ the fiber aspect ratio, $l$ the fiber length, and $\mathrm{v}_{f}$ the fiber volume fraction. There are two empirical parameters, which need to be determined: $\mathrm{v}_{c}$ is a critical volume fraction, and $n$ is an exponent index. Both $\mathrm{v}_{\mathcal{C}}$ and $n$ can determine the slope and upper boundary of this empirical function. We believe the correcting coefficient $d_{c}$ is a function of both fiber volume content and fiber length. At very low concentration (dilute concentration), the fibers have a much less chance to contact with each other and form bundles. The value of $d_{c}$ should approach 1.0 at low fiber volume content. In addition, $d_{c}$ should also keep increasing with $\mathrm{v}_{f}$ until reach an upper boundary. So, we modified the form of the logistic function or logistic curve ('S' shape) and proposed our empirical model in the form of Equation (10) [33].
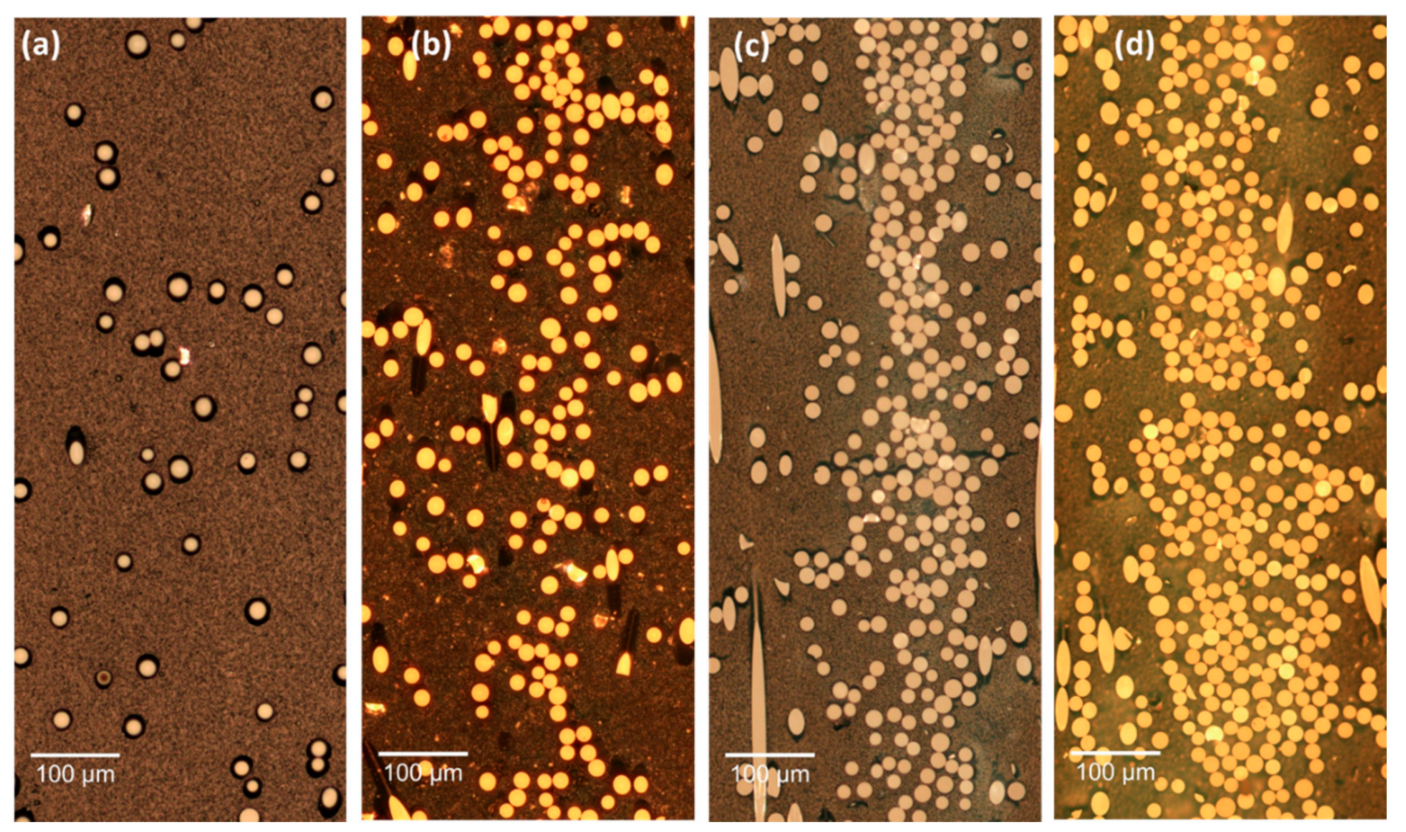

Figure 7. Cross-sectional microscopic images at the $r$ - $z$ plane for (a) $10 \mathrm{wt} \%$, (b) $30 \mathrm{wt} \%$, (c) $40 \mathrm{wt} \%$, and (d) $50 \mathrm{wt} \%$ glass fiber polypropylene composites.

This empirical model was used to correct the bundles' size and fit both HT and EMT models to the tensile test results in the Hele-Shaw region with the non-linear least squares fitting method. The measured FLD and experimental fourth order orientation tensor were also used in the calculation. The empirical parameters of the $d_{c}$ obtained by the fitting of both HT and EMT models are shown in Table 2. The comparisons of the fitted results with experiments at the Hele-Shaw region is shown in Figure 8a. After the application of the empirical model, the predictions turns out to be much more accurate when compared with tensile test results. However, this model might over-predict the bundles' size, because the perfect adhesion between glass fibers and matrix are assumed which might not be 
true. The empirical parameters obtained from the Hele-Shaw region were applied to calculate the modulus at the advancing-front. The comparisons among the as-calculated predictions, corrected predictions, and the experimental results are shown in Figure $8 \mathrm{~b}$. The predictions also show significant improvement after the diameter correction.

Table 2. Fitted parameters of the empirical model.

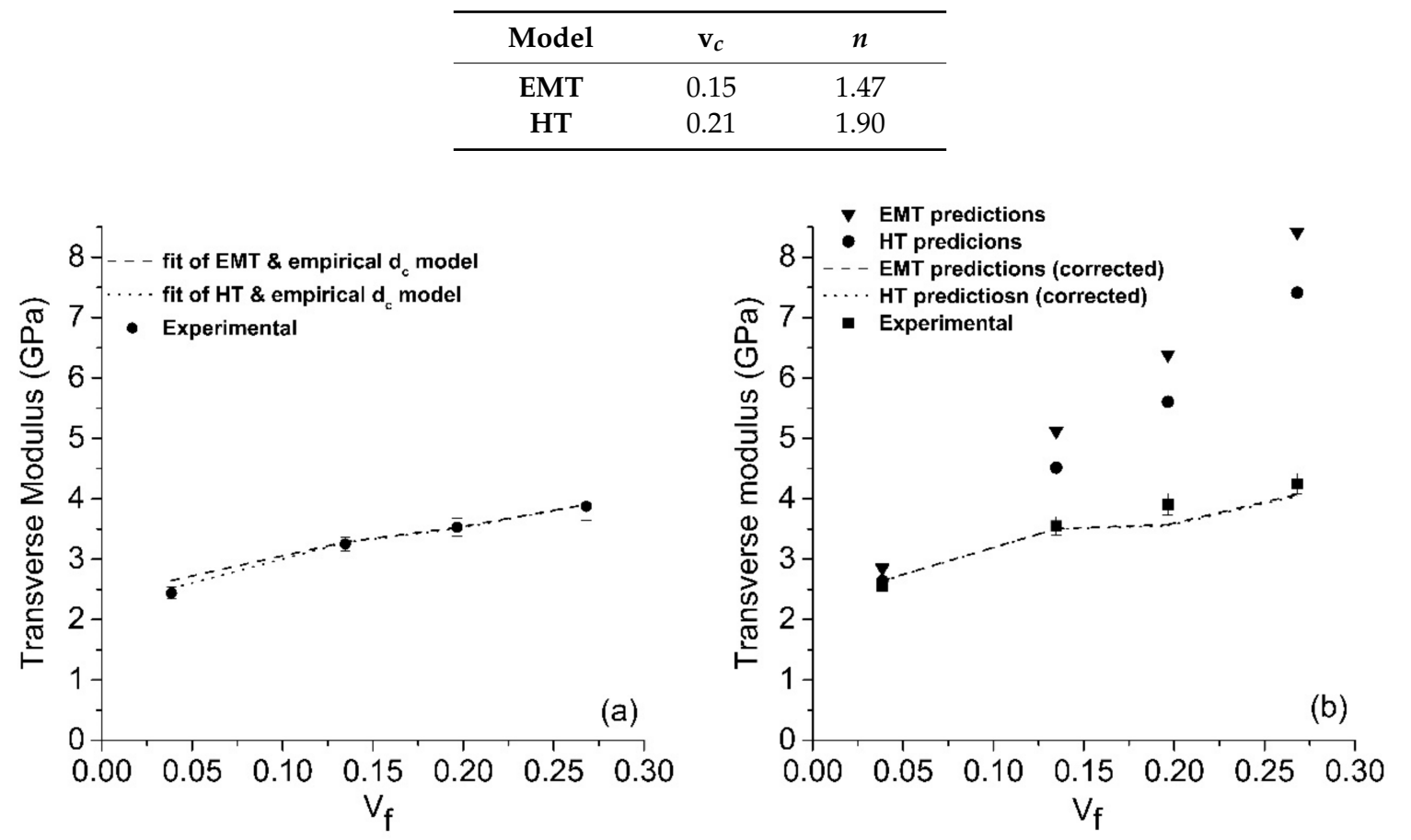

Figure 8. A comparison between the fit of EMT and HT with the experimental tensile test results with various fiber content, at the (a) Hele-Shaw region. Comparisons among the as-calculated predictions, the corrected predictions using the fitted parameters from Hele-Shaw region and experimental data at (b) the advancing front region.

Figure 9 shows the fiber bundles' size as a function of fiber length at various fiber concentration using Equation (10). The two empirical parameters were obtained by fitting the predictions from the EMT method to experimental tensile data. At low concentration (10 wt $\%$ ), the magnitude of $d_{c}$ barely increased in the length range from $0.06 \mathrm{~mm}$ to $12 \mathrm{~mm}$. The slopes of the lines increase notably with concentration. At higher concentration $(30,40,50 \mathrm{wt} \%)$ the values of $d_{c}$ show a rapid increase with fiber length. The trends of $d_{c}$ can be explained qualitatively from the fiber breakage aspect. At higher fiber content, the dominant fiber breakage mechanisms are fiber-fiber and fiber-machine interactions [34]. The residual fiber length exhibited a linear decrease following an increase in the fiber content $[14,35]$. Several authors also suggested that the fiber breakage rate was proportional to the fiber length or fiber aspect ratio [12,36]. The calculated bundle size, $d_{c}$, for those longer fibers is very large, especially for the $50 \mathrm{wt} \%$ fiber content. Qualitatively, under high fiber content, those long fibers have a great chance to contact the neighboring fibers and the machine (screw and wall of barrel). Fiber bundles reduce the effective fiber length or aspect ratio, significantly, allowing the preservation of longer fibers for higher content fiber composites during the intensive injection molding process. 


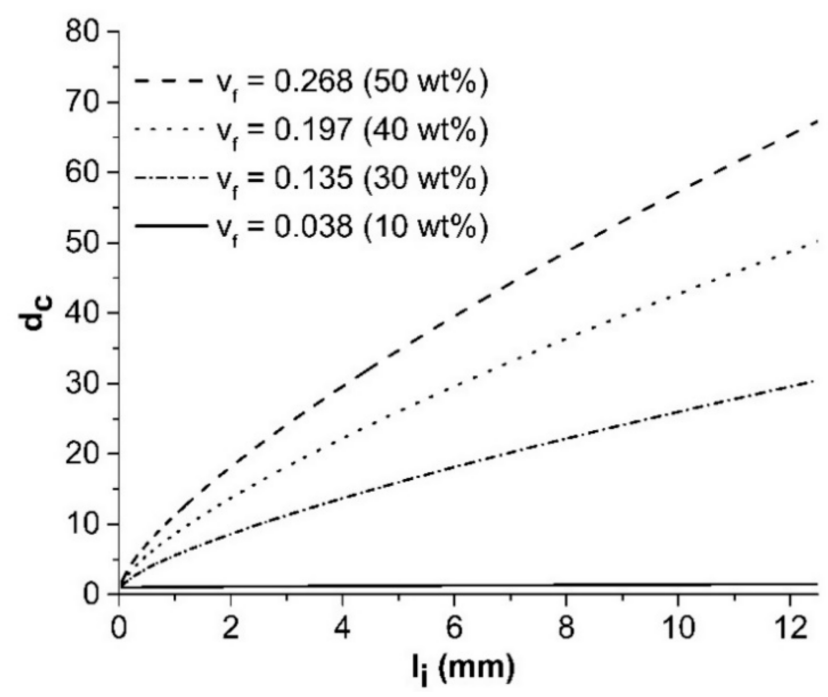

Figure 9. Predicted fiber bundles' size as a function of fiber length with various fiber concentrations by fitting the predictions using EMT method to experimental tensile data.

\section{Conclusions}

The stiffness properties have been studied in this work for $10 \mathrm{wt} \%, 30 \mathrm{wt} \%, 40 \mathrm{wt} \%$, and $50 \mathrm{wt} \%$ injection molded LFTs (glass/PP). Experimental measurements of fiber length distribution and fiber orientation were obtained to calculate the transverse modulus ( $\theta$ direction) using both Halpin-Tsai (HT) model and the Mori-Tanaka model based on Eshelby's equivalent inclusion (EMT). It has been shown that the EMT method generates slightly larger values than does the HT model. The accuracy of the various closure approximations for predicting the elastic properties has also been evaluated. The IBOF and ORW3 approximations turned out to be the best ways of describing the fourth order tensor in terms of the second order tensor. An important finding from this work is that the models over-predicted the modulus for higher fiber content injection molded LFTs. An empirical model was developed to correct the fibers' aspect ratio in the computations obtained for the actual as-formed LFTs with fiber bundles under high fiber content. After the correction, the analytical predictions matched well with the experimental stiffness values from tensile tests on the LFTs. The leveling off of the elastic properties of the injection molded LFTs as fiber content increases $(>30 \mathrm{wt} \%)$ is due to the existence of the fiber bundles. In this study, we assume the adhesion is perfect to simplify the problem, which most likely not be true. However, the $10 \mathrm{wt} \%, 30 \mathrm{wt} \%, 40 \mathrm{wt} \%$, and $50 \mathrm{wt} \%$ materials have the same surface treatments for the fibers. Therefore, it is legitimate to only include the effects of the clumping of fibers for comparison purpose. Moreover, the calculated bundle sizes from the proposed empirical model can be further applied to predict the strength in future work.

Author Contributions: H.C. performed the work on the fiber orientation, fiber length analysis, and tensile test. H.C. also did all the elastic properties predictions and the development of the proposed empirical bundles' size model. D.G.B. had oversight in all aspects of the project and were involved in all stages of the research.

Acknowledgments: We would like to thank the SABIC Innovative Plastics for supplying all the materials in this study. The authors would also like to thank the Material Science and Engineering department and the Sustainable Energy laboratory at Virginia Tech for the use of laboratory equipment. This work was funded, in part, by the American Chemical Council.

Conflicts of Interest: The authors declare no conflict of interest. The sponsors had no role in the design of the study; in the collection, analyses, or interpretation of data; in the writing of the manuscript, and in the decision to publish the results. 


\section{References}

1. Henning, F.; Ernst, H.; Brüssel, R. Lfts for automotive applications. Reinf. Plast. 2005, 49, 24-33. [CrossRef]

2. Ortman, K.; Baird, D.; Wapperom, P.; Whittington, A. Using startup of steady shear flow in a sliding plate rheometer to determine material parameters for the purpose of predicting long fiber orientation. J. Rheol. 2012, 56, 955-981. [CrossRef]

3. Von Turkovich, R.; Erwin, L. Fiber fracture in reinforced thermoplastic processing. Polym. Eng. Sci. 1983, 23, 743-749. [CrossRef]

4. Advani, S.G.; Tucker, C.L., III. The use of tensors to describe and predict fiber orientation in short fiber composites. J. Rheol. 1987, 31, 751-784. [CrossRef]

5. Garesci, F.; Fliegener, S. Young's modulus prediction of long fiber reinforced thermoplastics. Compos. Sci. Technol. 2013, 85, 142-147. [CrossRef]

6. Tucker Iii, C.L.; Liang, E. Stiffness predictions for unidirectional short-fiber composites: Review and evaluation. Compos. Sci. Technol. 1999, 59, 655-671. [CrossRef]

7. Hine, P.J.; Rudolf Lusti, H.; Gusev, A.A. Numerical simulation of the effects of volume fraction, aspect ratio and fibre length distribution on the elastic and ther moelastic properties of short fibre composites. Compos. Sci. Technol. 2002, 62, 1445-1453. [CrossRef]

8. Ingber, M.S.; Papathanasiou, T.D. A parallel-supercomputing investigation of the stiffness of aligned, short-fiber-reinforced composites using the boundary element method. Int. J. Numer. Methods Eng. 1997, 40, 3477-3491. [CrossRef]

9. Takao, Y.; Taya, M. The effect of variable fiber aspect ratio on the stiffness and thermal expansion coefficients of a short fiber composite. J. Compos. Mater. 1987, 21, 140-156. [CrossRef]

10. Halpin, J.C.; Jerine, K.; Whitney, J.M. The laminate analogy for 2 and 3 dimensional composite materials. J. Compos. Mater. 1971, 5, 36-49. [CrossRef]

11. Hine, P.J.; Lusti, H.R.; Gusev, A.A. On the possibility of reduced variable predictions for the thermoelastic properties of short fibre composites. Compos. Sci. Technol. 2004, 64, 1081-1088. [CrossRef]

12. Bumm, S.H.; White, J.L.; Isayev, A.I. Glass fiber breakup in corotating twin screw extruder: Simulation and experiment. Polym. Compos. 2012, 33, 2147-2158. [CrossRef]

13. Thomason, J.L. The influence of fibre length and concentration on the properties of glass fibre reinforced polypropylene. 6 . The properties of injection moulded long fibre pp at high fibre content. Compos. Part A Appl. Sci. Manuf. 2005, 36, 995-1003. [CrossRef]

14. Thomason, J.L. Structure-property relationships in glass-reinforced polyamide, part 1: The effects of fiber content. Polym. Compos. 2006, 27, 552-562. [CrossRef]

15. Halpin, J. Stiffness and expansion estimates for oriented short fiber composites. J. Compos. Mater. 1969, 3, 732-734. [CrossRef]

16. Affdl, J.; Kardos, J. The halpin-tsai equations: A review. Polym. Eng. Sci. 1976, 16, 344-352. [CrossRef]

17. Hill, R. A self-consistent mechanics of composite materials. J. Mech. Phys. Solids 1965, 13, 213-222. [CrossRef]

18. Dray, D.; Gilormini, P.; Régnier, G. Comparison of several closure approximations for evaluating the thermoelastic properties of an injection molded short-fiber composite. Compos. Sci. Technol. 2007, 67, 1601-1610. [CrossRef]

19. Taya, M.; Mura, T. On stiffness and strength of an aligned short-fiber reinforced composite containing fiber-end cracks under uniaxial applied stress. J. Appl. Mech. 1981, 48, 361-367. [CrossRef]

20. Camacho, C.W.; Tucker, C.L.; Yalvaç, S.; McGee, R.L. Stiffness and thermal expansion predictions for hybrid short fiber composites. Polym. Compos. 1990, 11, 229-239. [CrossRef]

21. Hyer, M.W. Stress Analysis of Fiber-reinforced Composite Materials; DEStech Publications Inc.: Lancaster, PA, USA, 2009.

22. Standard, A. Standard Test Method for Tensile Properties of Polymer Matrix Composite Materials. Available online: http:/ / file.yizimg.com/175706/2012061422194947.pdf (accessed on 6 August 2018).

23. Kunc, V.; Frame, B.J.; Nguyen, B.N.; Tucker, C.L., III; Velez-Garcia, G. Fiber Length Distribution Measurement for Long Glass and Carbon Fiber Reinforced Injection Molded Thermoplastics. Available online: https:/ / www.researchgate.net/profile/Gregorio_Velez-Garcia/publication/237431694_ FIBER_LENGTH_DISTRIBUTION_MEASUREMENT_FOR_LONG_GLASS_AND_CARBON_FIBER_ REINFORCED_INJECTION_MOLDED_THERMOPLASTICS/links/00b49531712e6067b3000000/ 
FIBER-LENGTH-DISTRIBUTION-MEASUREMENT-FOR-LONG-GLASS-AND-CARBON-FIBERREINFORCED-INJECTION-MOLDED-THERMOPLASTICS.pdf (accessed on 3 August 2018).

24. Vélez-García, G.; Wapperom, P.; Kunc, V.; Baird, D.; Zink-Sharp, A. Sample preparation and image acquisition using optical-reflective microscopy in the measurement of fiber orientation in thermoplastic composites. J. Microsco. 2012, 248, 23-33. [CrossRef] [PubMed]

25. Vélez-García, G.M.; Wapperom, P.; Baird, D.G.; Aning, A.O.; Kunc, V. Unambiguous orientation in short fiber composites over small sampling area in a center-gated disk. Compos. Part A Appl. Sci. Manuf. 2012, 43, 104-113. [CrossRef]

26. Chung, D.H.; Kwon, T.H. Improved model of orthotropic closure approximation for flow induced fiber orientation. Polym. Compos. 2001, 22, 636-649. [CrossRef]

27. Chung, D.H.; Kwon, T.H. Invariant-based optimal fitting closure approximation for the numerical prediction of flow-induced fiber orientation. J. Rheol. 2002, 46, 169-194. [CrossRef]

28. Houshyar, S.; Shanks, R.A.; Hodzic, A. The effect of fiber concentration on mechanical and thermal properties of fiber-reinforced polypropylene composites. J. Appl. Polym. Sci. 2005, 96, 2260-2272. [CrossRef]

29. Little, J.E.; Yuan, X.; Jones, M.I. Characterisation of voids in fibre reinforced composite materials. NDT E Int. 2012, 46, 122-127. [CrossRef]

30. Lee, D.J.; Oh, H.; Song, Y.S.; Youn, J.R. Analysis of effective elastic modulus for multiphased hybrid composites. Compos. Sci. Technol. 2012, 72, 278-283. [CrossRef]

31. Swolfs, Y.; Gorbatikh, L.; Romanov, V.; Orlova, S.; Lomov, S.V.; Verpoest, I. Stress concentrations in an impregnated fibre bundle with random fibre packing. Compos. Sci. Technol. 2013, 74, 113-120. [CrossRef]

32. Pratten, N.A. The precise measurement of the density of small samples. J. Mater. Sci. 1981, 16, $1737-1747$. [CrossRef]

33. Berger, R. Comparison of the gompertz and logistic equations to describe plant disease progress. Phytopathology 1981, 71, 716-719. [CrossRef]

34. Richard, V.T.; Lewis, E. Fiber fracture in reinforced thermoplastic processing. Polym. Eng. Sci. 1983, 23, 743-749.

35. Cieslinski, M.J.; Wapperom, P.; Baird, D.G. Influence of fiber concentration on the startup of shear flow behavior of long fiber suspensions. J. Non-Newton. Fluid Mech. 2015, 222, 163-170. [CrossRef]

36. Phelps, J.H.; Abd El-Rahman, A.I.; Kunc, V.; Tucker, C.L. A model for fiber length attrition in injection-molded long-fiber composites. Compos. Part A Appl. Sci. Manuf. 2013, 51, 11-21. [CrossRef] 